\title{
A Case Report, Evans Syndrome on Female Adult
}

\author{
Anak Agung Made Sedana Putra ${ }^{\mathrm{a}}$, Paulus B. Notopuro ${ }^{\mathrm{b}}$ \\ asedana2105@gmail.com \\ ${ }^{a}$ Resident of Clinical Pathology Department, Faculty of Medicine Airlangga University, Dr. Soetomo Hospital, Surabaya, Indonesia \\ ${ }^{b}$ Department of Clinical Pathology, Faculty of Medicine Airlangga University, Dr. Soetomo Hospital, Surabaya, Indonesia
}

\begin{abstract}
Background: Evans syndrome (ES) is a rare and chronic autoimmune disease characterized by autoimmune hemolytic anemia (AIHA) and immune thrombocytopenic purpura (ITP) with a positive direct anti-human globulin test. Evan's syndrome is a rare disorder because it is diagnosed in only $0.8 \%$ to $3.7 \%$ of all patients with either ITP or AIHA at onset. The clinical presentation can include fatigue, pallor, jaundice and mucosal bleeding, with remissions and exacerbations during the person's lifetime, and acute manifestations as catastrophic bleeding and massive hemolysis.

Case Description: A 20-year-old female was referred from Mardi Waluyo Hospital with complaints of weakness since 1 week before admission. She also complained of pallor, headache and gum bleeding. Physical examination: temperature $36.2^{\circ} \mathrm{C}$, heart rate $90 \mathrm{x} / \mathrm{minute}$, respiratory rate $18 \mathrm{x} /$ minute and blood pressure $120 / 70 \mathrm{mmHg}$, without organ enlargement. Laboratory result: Hemoglobin 5.6 g/dL, WBC $5.03 \times 103 / \mu \mathrm{L}$, Platelets $62.0 \times 103 / \mu \mathrm{L}, \mathrm{C} 359.5, \mathrm{C} 416.8$, positive direct and indirect Coomb's test, direct bilirubin $0.46 \mathrm{mg} / \mathrm{dL}$, total bilirubin $3.61 \mathrm{mg} / \mathrm{dL}$. Blood Smear: normochromic anisopoikilocytosis anemia and thrombocytopenia. BONE MARROW ASPIRATION: Increase in erythropoietic activity, increase in thrombopoietic activity.

Conclusion: Based on the physical examination, laboratory results especially positive direct Coomb's test and increase in marrow's erythropoietic and thrombopoietic activity, the cytopenia in this patient was due to peripheral destruction factor, possibly an autoimmune disease. This patient fulfilled the diagnosis of Evans Syndrome, although it is needed to be confirmed by antiplatelet antibody.
\end{abstract}

Key words: Evans Syndrome, AIHA, ITP.

\section{Introduction}

Evans syndrome is a rare and chronic autoimmune disease, characterized by autoimmune hemolytic anemia (AIHA) and immune thrombocytopenic purpura (ITP) with positive direct anti-human globulin results. ${ }^{1}$ AIHA is the most common hemolytic autoimmune disease. Antibodies attached to red blood cells are recognized and carried by macrophages in the spleen. These cells then phagocytize part of the red blood cell membrane (RBC), which then causes a change in shape to become spherocytes. Spherocytes do not have the flexibility of normal $\mathrm{HR}$, thus facilitating their destruction in the reticuloendothelial system, leading to extravascular hemolysis. ITP is a condition where the platelet count is low due to an autoimmune process of antibodies against platelets. The presence of antibodies attached to platelets makes them susceptible to opsonization and phagocytosis by macrophages in the spleen. ${ }^{2}$

Evans syndrome is said to be a rare disease because it is only seen in $0.8-3.7 \%$ of patients with an initial diagnosis of AIHA and ITP. There is not much literature on evan syndrome, and most of the literature is on pediatric patients so that the characteristics and outcomes of evans syndrome patients in adults are not widely known. ${ }^{3}$

Clinical symptoms may include weakness, pallor, jaundice and mucosal bleeding. Remissions and exacerbations can occur during the patient's life, with acute manifestations of bleeding and severe hemolysis. ${ }^{1}$

\section{Case presentation}

Female, 20 years old, referred from Mardi Waluyo Hospital with the main complaint of feeling weak since 1 week before being admitted to the hospital. The patient also complains of pallor, headache and bleeding gums. Urination of normal color and frequency, bowel movements of normal color and consistency. Normal menstruation. The patient was treated at the Mardi Waluyo Hospital for 2 days with the reason for the decrease in hemoglobin and platelets, but the patient had not received a transfusion because there was no suitable donor. Physical examination: temperature $36.2^{\circ} \mathrm{C}$, heart rate $90 \mathrm{x} /$ minute, respiratory rate $18 \mathrm{x} / \mathrm{minute}$ and blood pressure $120 / 70 \mathrm{mmHg}$, anemic and icteric sclera without organ enlargement. Laboratory result: Hemoglobin 5.6 g/dL, WBC 5.03 x $103 / \mu \mathrm{L}$, Platelets $62.0 \times 10^{3} / \mu \mathrm{L}$, IRF 37\%, Reticulocyte 32,28\% and IPF 9\% C3 59.5, C4 16.8, positive direct and indirect Coomb's test, direct bilirubin $0.46 \mathrm{mg} / \mathrm{dL}$, total bilirubin $3.61 \mathrm{mg} / \mathrm{dL}$. There were an increase in hemoglobin and platelets to $6.1 \mathrm{~g} / \mathrm{dL}$ and $138 \times 10^{3} / \mu \mathrm{L}$ respectively, also decrease in reticulocyte to $22,8 \%$ and IPF to $5.7 \%$ after the administration of methylprednisolone $125 \mathrm{mg} / 8$ hours. Blood Smear: normochromic anisopoikilocytosis anemia and thrombocytopenia. Bone marrow aspiration: Increase in erythropoietic activity, 
increase in thrombopoietic activity. Other examination include ANA test and HbsAg with negative result, The patient was then diagnosed as suspected Evans syndrome.
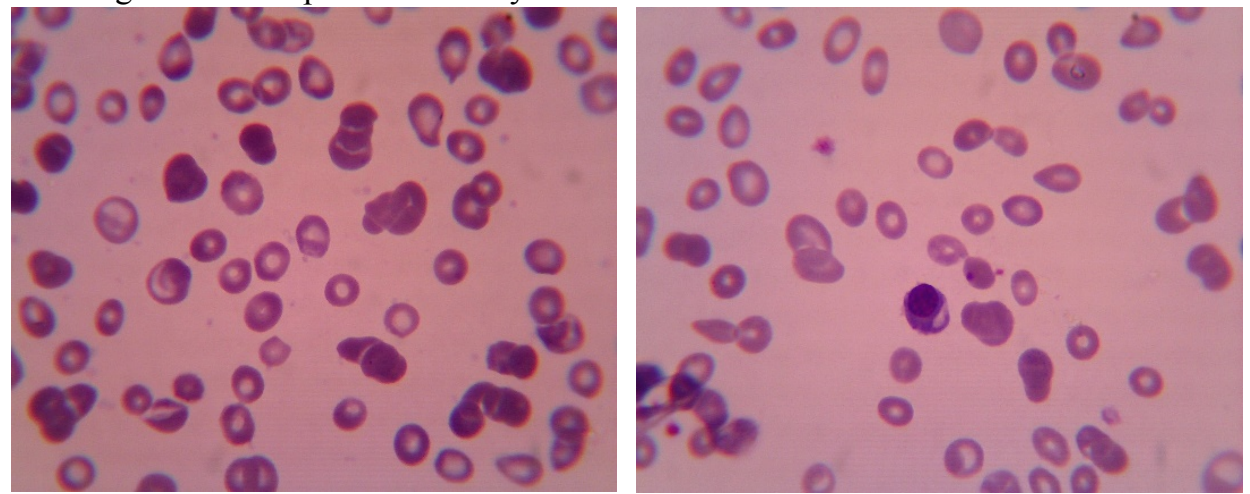

Figure 1. There is agglutination (left), the impression of a decreased number of platelets (right)
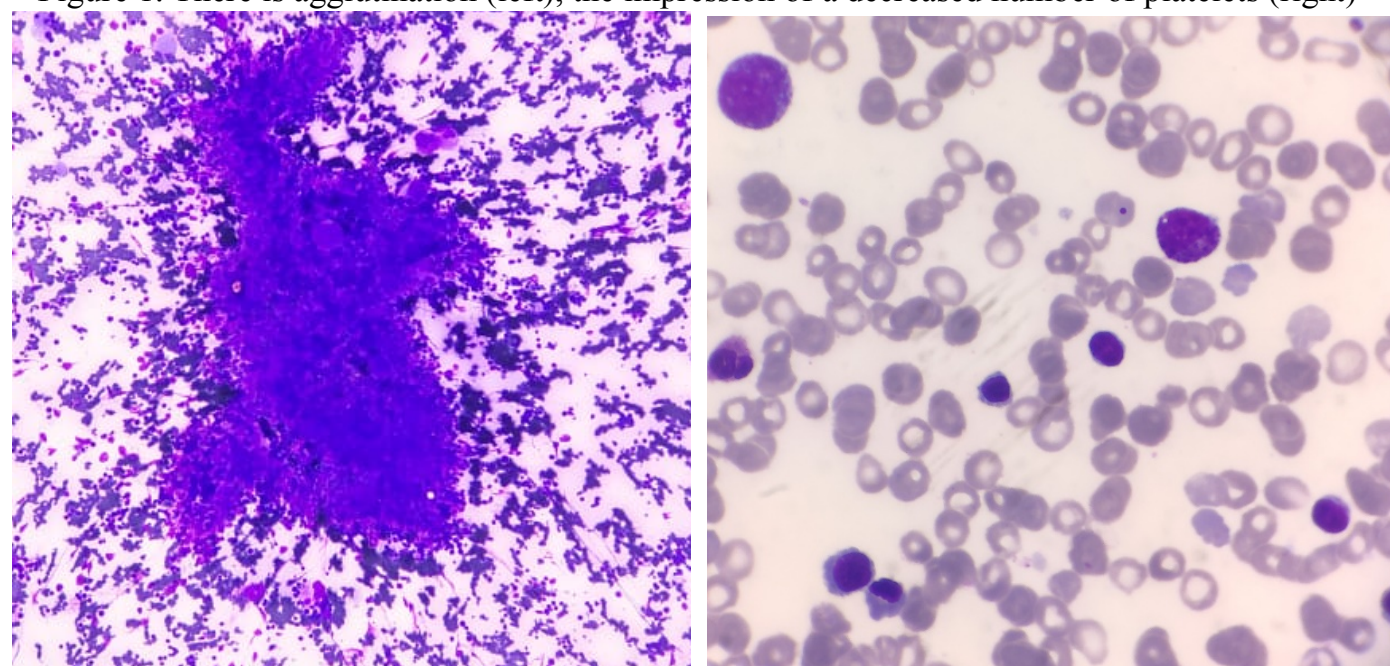

Figure 2. Bone marrow aspiration picture, shows increased cellularity (left), and agglutination and increased erythropoiesis (right)

\section{3.. Discussion}

Evans syndrome is a rare and chronic autoimmune disease, characterized by the presence of AIHA and ITP with positive direct anti-human globulin results. ${ }^{1}$

The diagnosis of AIHA is made based on the presence of anemia, signs of hemolysis with reticulocytosis, low haptopglobin values, elevated lactate dehydrogenase levels, elevated indirect bilirubin levels, and a positive anti-human globulin test (Coomb's test). These signs are sometimes not always found in cases of AIHA. AIHA can be caused by warm antibodies or cold antibodies. ${ }^{4}$

This patient had symptoms of anemia in the form of weakness, paleness and headache with decreased hemoglobin. The signs of hemolysis in this patient are the presence of spherocytes and polychromatic cells that can be found on peripheral blood smear examination. The patient also had jaundice with an increase in total and indirect bilirubin levels. The results of the direct, indirect and auto control Coomb tests in this patient were positive. BONE MARROW examination with results of increased erythropoiesis activity, results of 32.28\% reticulocytes and increased IRF (37\%) and a positive Coomb test indicated the presence of antibodies against red blood cells that caused destruction of red blood cells in the circulation. These results indicate that the patient fulfills the diagnosis of AIHA.

Diagnosis of ITP according to the 2011 Clinical Practice Guideline on the Evaluation and Management of Immune Thrombocytopenia (ITP), namely: the presence of bleeding consistent with thrombocytopenia without constitutional symptoms, on physical examination no hepatosplenomegaly, lymphadenopathy, and congenital stigmata, thrombocytopenia (platelet count $<100,000 / \mu 1$ ), as well as the results of blood smear examination with normal or large sized platelets. Bone marrow examination is performed if abnormalities are found in the history, physical examination, results of a complete blood count, and blood smear examination. ${ }^{5}$

In this patient, there were complaints of bleeding in the gums, on physical examination there was no hepatosplenomegaly, and thrombocytopenia. The blood smear examination results did not reveal any morphological abnormalities of platelets. Bone marrow examination with results of increased thrombopoiesis 
activity showed destruction in the circulation. The destruction of platelets in the circulation can be caused by the presence of antibodies against platelets but still requires confirmation by examination of antiplatelet antibodies. Thrombocytopenia can also be caused by ineffective thrombopoiesis. Ineffective thrombopoiesis can occur because antibodies that bind to megakaryocytes interfere with the production or release of platelets, it can also be due to antibody-mediated platelet phagocytosis in the endoplasmic reticulum of the bone marrow. ${ }^{6}$ Platelet yield increased 3 days after administration of methylprednisolone indicating a response to first-line ITP therapy. ${ }^{7}$ These results indicate that the patient fulfills the diagnosis of ITP.

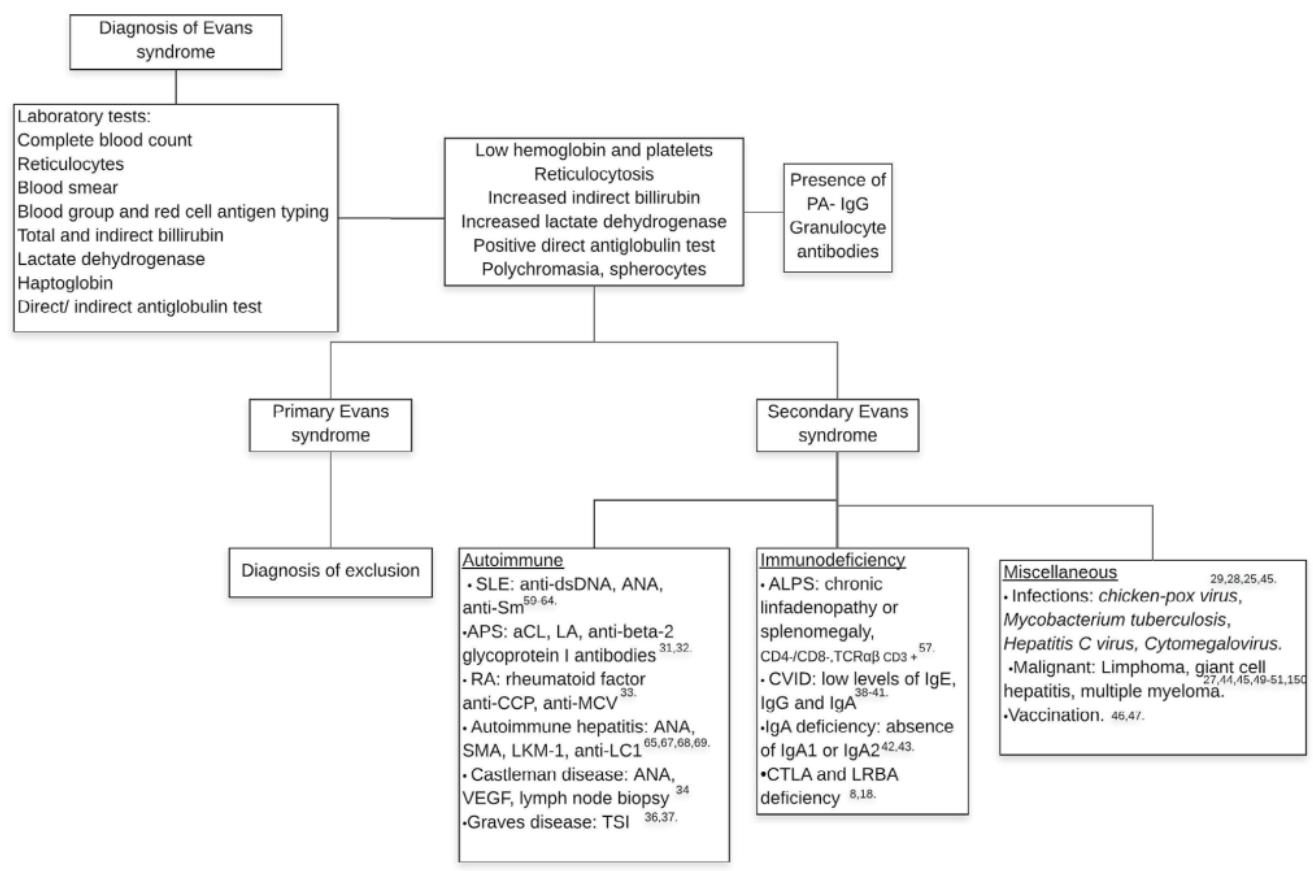

Figure 3. Evans Syndrome Diagnostic Approach.

Caption: aCL, anticardiolipin; ALPS, autoimmune lymphoproliferative syndrome; ANA, antinuclear antibodies; anti-CCP, anti-cyclic citrullinated peptide; antiLC1, anti-liver cytosol antibody; anti-MCv, anti-mutated citrullinated vimetin; anti-Sm, anti-Smith antibodies; APS, antiphospholipid syndrome; CVD, common variable immunodeficiency; LA, lupus anticoagulant; LKM-1, liver kidney microsomal type 1 antibodies; PA-IgG, platelet-associated IgG; RA, rheumatoid arthritis; SLE, systemic lupus erythematosus; SMA, smooth muscle antibody; TSi, thyroid stimulating immunoglobulin; veGF, vascular endothelial growth factor.1

Tracing clinical history to determine risk factors for Evans syndrome, such as: infection, malignancy, autoimmune disease, vaccination, medications and family history of autoimmune disease. Primary Evans syndrome is a diagnosis based on exclusion (figure 3), while in secondary Evans syndrome it is necessary to know the underlying disease, because of differences in therapy for primary and secondary Evans syndrome. ${ }^{1}$

This patient underwent an ANA examination with a negative result, to rule out autoimmune diseases such as SLE. The results of bone marrow examination showed increased erythropoiesis and thrombopoiesis, no dysplasia, signs of hematological malignancy, or non-hematological cell infiltration were found.

Management of Evan's syndrome is still a challenge, where there are periods of remission and exacerbation, as well as variations in response to therapy even in the same individual. First-line therapy for Evan's syndrome includes corticosteroids and intravenous immunoglobulin (IVIG) which responds to the majority of patients. Second-line therapy includes the immunosuppressive drugs ciclosporin, vincristine, danazol or a combination of these drugs. Recently, rituximab has been used for the management of autoimmune diseases. ${ }^{8}$

The patient received methylprednisolone therapy which is the first line therapy. After the patient received methylprednisolone therapy, the patient's hemoglobin yield increased from $5.5 \mathrm{~g} / \mathrm{dL}$ to $6.1 \mathrm{~g} / \mathrm{dL}$, and the platelet yield increased from $62,000 / \mu \mathrm{L}$ to $138,000 / \mu \mathrm{L}$. This indicates the patient is responding to therapy.

\section{Conclusion}

Based on the results of physical examination and laboratory results, especially positive Coomb test results and increased erythropoiesis and bone marrow thrombopoiesis activity, cytopenia in this patient could be due to peripheral destructive factors, possibly autoimmune disease. This patient fulfills the diagnosis of Evans Syndrome, but an antiplatelet antibody test is still required. 


\section{Acknowledgement}

The author would like to thank Mr. Paulus Budiono Notopuro, M.D., Ph.D., who has helped and guided the writing of these case report.

\section{References}

1. Jaime-Pérez J, Aguilar-Calderón P, Salazar-Cavazos L, Gómez-Almaguer D. Evans syndrome: clinical perspectives, biological insights and treatment modalities. Journal of Blood Medicine. 2018; Volume 9:171-84.

2. Yoshida H, Ishida H, Yoshihara T, Oyamada T, Kuwana M, Imamura T, et al. Complications of Evans' syndrome in an infant with hereditary spherocytosis: A case report. Journal of Hematology and Oncology. 2009;2:40.

3. Dave P, Krishna K, Diwan AG. Evan's syndrome revisited. The Journal of the Association of Physicians of India. 2012;60:60-1.

4. Valent P, Lechner K. Diagnosis and treatment of autoimmune haemolytic anaemias in adults: A clinical review. Wiener Klinische Wochenschrift. 2008;120:136-51.

5. Neunert C, Lim W, Crowther M, Cohen A, Solberg L, Crowther MA, et al. The American Society of Hematology 2011 evidence-based practice guideline for immune thrombocytopenia. Blood. 2011;117:4190-207.

6. Kuter DJ, Gernsheimer TB. Thrombopoietin and Platelet Production in Chronic Immune Thrombocytopenia. Hematology/Oncology Clinics of North America. 2009;23:1193-211.

7. Neunert CE. Management of newly diagnosed immune thrombocytopenia: can we change outcomes? Blood Advances. 2017;1:2295-301.

8. Norton A, Roberts I. Management of Evans syndrome. British Journal of Haematology. 2006;132:12537. 